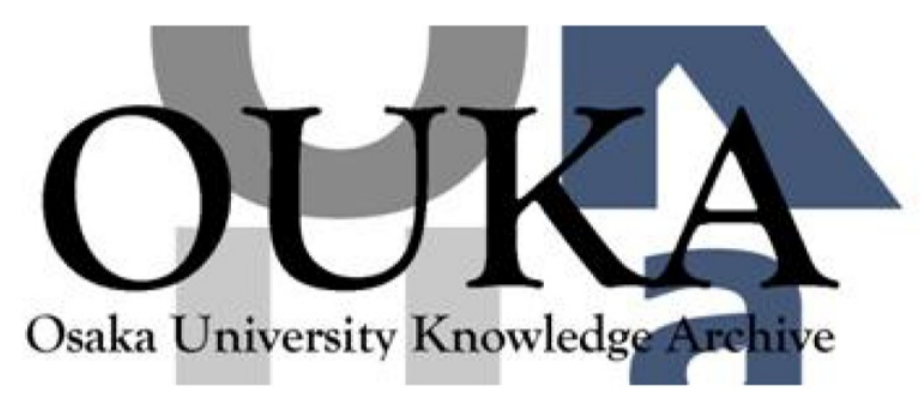

\begin{tabular}{|c|l|}
\hline Title & $\begin{array}{l}\text { Enhancement of third-order optical } \\
\text { nonlinearities by conjugated polymer-bonded } \\
\text { carbon nanotubes }\end{array}$ \\
\hline Author(s) & Feng, Wei; Yi, Wenhui; Wu, Hongcai et al. \\
\hline Citation & $\begin{array}{l}\text { Journal of Applied Physics. 98(3) p. 034301- } \\
\text { p. } 034301\end{array}$ \\
\hline Issue Date & $2005-08-03$ \\
\hline oaire:version & VoR \\
\hline URL & https://hdl. handle.net/11094/75848 \\
\hline rights & \\
\hline Note & \\
\hline
\end{tabular}

Osaka University Knowledge Archive : OUKA

https://ir. Library. osaka-u. ac. jp/

Osaka University 


\section{Enhancement of third-order optical nonlinearities by conjugated polymer- bonded carbon nanotubes}

Cite as: J. Appl. Phys. 98, 034301 (2005); https://doi.org/10.1063/1.1954887

Submitted: 01 February 2005. Accepted: 20 May 2005. Published Online: 03 August 2005

Wei Feng, Wenhui Yi, Hongcai Wu, Masanori Ozaki, and Katsumi Yoshino

\section{ARTICLES YOU MAY BE INTERESTED IN}

Third-order optical nonlinearities of carbon nanotubes in the femtosecond regime Applied Physics Letters 85, 3572 (2004); https://doi.org/10.1063/1.1808226

Ultrafast optical switching properties of single-wall carbon nanotube polymer composites at $1.55 \mu \mathrm{m}$

Applied Physics Letters 81, 975 (2002); https://doi.org/10.1063/1.1498007

The third-order optical nonlinearities of carbon nanotube modified conjugated polymer in the femtosecond and nanosecond regimes

Journal of Applied Physics 100, 094301 (2006); https://doi.org/10.1063/1.2363550

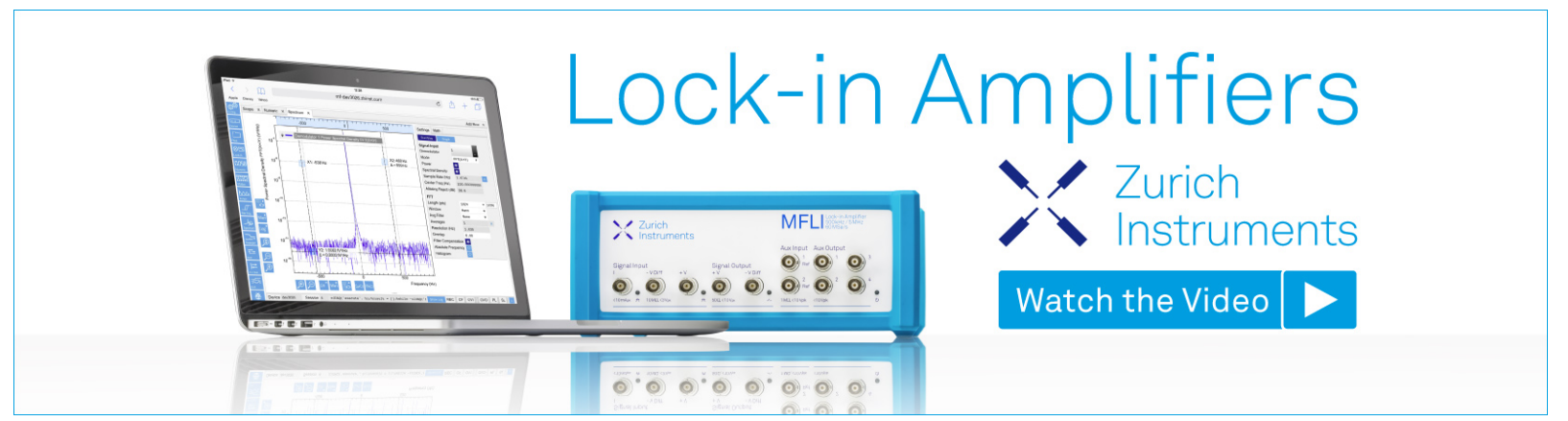




\title{
Enhancement of third-order optical nonlinearities by conjugated polymer-bonded carbon nanotubes
}

\author{
Wei Feng ${ }^{\text {a) }}$ \\ School of Materials Science and Engineering, Tianjin University, 300072 Tianjin China \\ Wenhui Yi and Hongcai Wu \\ School of Information and Electronics Engineering, 710049 Xi'an China \\ Masanori Ozaki and Katsumi Yoshino \\ Department of Electronic Engineering, Graduate School of Engineering, Osaka University, \\ 2-1 Yamada-Oka, Suita, Osaka 565-0871, Japan
}

(Received 1 February 2005; accepted 20 May 2005; published online 3 August 2005)

\begin{abstract}
Third-order nonlinear susceptibility $\chi^{(3)}$ and second-order hyperpolarizability $\gamma$ of poly\{[3-octylthiophene-2,5-diyl]-[p-aminobenzylidenequinomethane]-bonded multiwalled carbon nanotubes (POTABQ-MWNTs) were measured in chloroform solution using degenerate four-wave mixing at $532 \mathrm{~nm}$ under nanosecond pulse excitation. Effective nonlinear absorption coefficient $\beta_{\text {eff }}$ and nonlinear refraction index $n_{2}$ were obtained using open aperture and close aperture $Z$-scan technique at the same wavelength, respectively. Optical limiting property of the sample was also investigated. The resulting POTABQ-MWNTs exhibited large third-order nonlinear optical responses mainly due to the formation of intramolecular photoinduced charge-transfer system of polymers and carbon nanotubes. (C) 2005 American Institute of Physics. [DOI: 10.1063/1.1954887]
\end{abstract}

\section{INTRODUCTION}

Nonlinear optical (NLO) materials are required in a wide range of important applications, such as optical limiting, optical computing, and optical communication. Efforts are in progress in many laboratories for the realization of all optical devices. Such devices can do away with electrical/optical/ electrical conversion existing in the present communication system, which really limits the optimum use of available speed. $^{1,2}$ In the light of wide applications of NLO effects, a large number of materials have been synthesized and their NLO properties have been explored.

In recent years, carbon nanotubes have been investigated for many potential applications. ${ }^{3,4}$ Their unique onedimensional $\pi$-electron conjugation, mechanical strength, and high thermal and chemical stabilities make them very attractive for use in many applications. Theoretically, the magnitude of the second-order hyperpolarizabilities $\gamma$ of single-walled carbon nanotubes (SWNTs) was estimated for $10^{-29}$ esu. $^{5-9}$ To date, only few experiments of the third-order optical nonlinearity have been carried out due to the poor solubility of carbon nanotubes. ${ }^{10-16}$ The third-order susceptibilities $\chi^{(3)}$ of multiwalled nanotubes (MWNTs) were first measured by using picosecond and nanosecond degenerate four-wave mixing (DFWM) techniques by Liu et al. ${ }^{10}$ The magnitude of picosecond $\chi^{(3)}$ and nanosecond $\chi^{(3)}$ was measured to be $10^{-14}$ and $10^{-11}$ esu, respectively. Wang et al. ${ }^{11}$ reported a femtosecond time-resolved third-order NLO investigation of the dispersion of shortened SWNTs in $N, N$-dimethylformamide (DMF). The average of magnitude of $\gamma$ for each SWNT was estimated to be $2.1 \times 10^{-28}$ esu. It

\footnotetext{
a) Author to whom correspondence should be addressed; FAX: +86-2227404724; electronic mail: 1f@xjtu.edu.cn
}

should be noted that the above investigations were made on carbon nanotubes dispersed in organic solvents due to their poor solubility, and such dispersions were unstable at high concentrations. Hence it is highly desirable to obtain stable solutions containing carbon nanotubes.

The recent discovery of photoinduced electron transfer from $\pi$-conjugated polymers onto fullerene to form photoinduced charge-transfer systems has generated considerable interest because of its implications in both pure and applied areas of research. ${ }^{17-20}$ Ultrasfast spectroscopic studies show that the initial charge transfer occurs within the first $300 \mathrm{fs}$ following excitation of the polymer and that the system quickly relaxes to a metastable with the electron on the fullerene and a positive charge on the polymer. Because of the nearly instantaneous response of the system to illumination and because of the tunability of the decay dynamics by fullerene concentration, conjugated polymer/fullerene blends are promising candidates for nonlinear optical applications. ${ }^{21}$

Moreover, polymers are attractive since they can be easily processed and fabricated into solid-state forms such as thin films. Both polymer-coated, such as polyethylene glycol (PEG)-, poly-2-vinylpyridine (P2VP)-, poly-4-vinylpyridine (P4VP)- and poly-4-vinylphenol (PVPh)-coated MWNTs, and polymer-bonded MWNTs, such as polyethylene oxide (PEO)-bonded MWNTs, were synthesized and their thirdorder NLO properties were measured by Jin et al., ${ }^{12}$ However, the polymers they employed were linear polymers and exhibited no meaningful NLO responses themselves.

Tang and $\mathrm{Xu}^{22}$ reported the preparation and the NLO properties of poly(pheylacetylene) (PPA)-wrapped carbon nanotubes (NT/PPA). The photoinduced charge-transfer process was characterized by fluorescence quenching. The resulting NT/PPA solutions exhibited strong optical limiting 


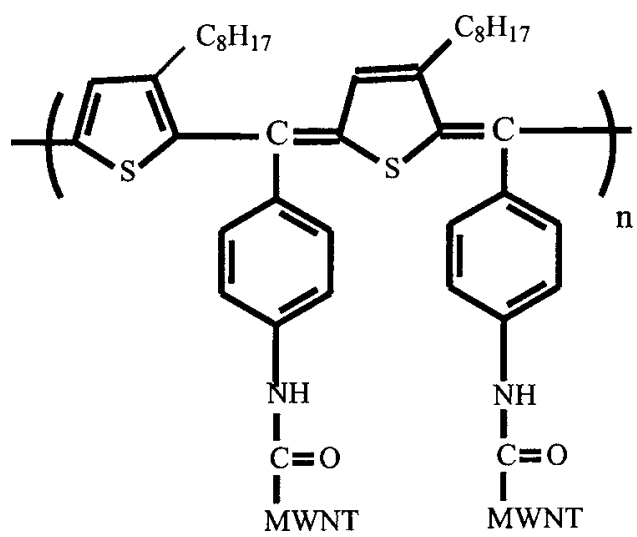

FIG. 1. Chemical structure of POTABQ-MWNTs.

properties at $532 \mathrm{~nm}$ arising from the NLO absorption of carbon nanotubes and PPA and scattering of carbon nanotubes. O'Flaherty et al. ${ }^{23}$ used poly(metavinylene-co-2,5dioctyloxy-para-phenylene-vinylene) (PmPV) as matrix to disperse MWNTs and found that the NLO absorption coefficients were enhanced at high MWNT concentrations probably due to tube-tube interaction and rise of charge delocalization. However, since the magnitude of third-order NLO susceptibility $\chi^{(3)}$ for PPA and PmPV was below $10^{-10}$ esu, the magnitude of $\chi^{(3)}$ for the resulting PPA/MWNT and PmPV/MWNT composites was not large enough to be used in the NLO applications yet.

According to the theoretical and experimental studies, polyheteroarylene methines exhibited extremely narrow band gap $\left(E_{g}=0.7 \mathrm{eV}\right)($ Refs. 24-26) and large third-order NLO responses $\left(\chi^{(3)}=10^{-9}-10^{-7} \mathrm{esu}\right) .^{27,28}$ In our previous study, a series of more than ten poly(heteroarylene methines) derivatives were synthesized ${ }^{29}$ and the third-order NLO properties were studied by using $Z$-scan, ${ }^{29} \mathrm{DFWM},{ }^{30}$ and time-resolved optical Kerr effect techniques (OKE) ${ }^{30}$ In this study, we report the third-order NLO properties of POTABQ-MWNTs. The third-order susceptibility $\chi^{(3)}$ and the second-order hyperpolarizability $\gamma$ of POTABQ-MWNTs are studied by using nanosecond DFWM technique. Nonlinear absorption coefficient $\beta_{\text {eff }}$ and nonlinear refraction index $n_{2}$ are studied by using open aperture and closed aperture $Z$-scan techniques, respectively. Optical limiting property of the sample is also investigated.

\section{EXPERIMENTAL SECTION}

POTABQ-MWNTs were synthesized via amidiation reactions. The synthesis and characterization of this material were published elsewhere. ${ }^{31}$ Figure 1 shows the molecular structure of POTABQ-MWNTs. All the solvents and chemicals were analyzing reagent grade and were obtained from Beijing Chem. Inc.

The DFWM experiment was carried out at $532 \mathrm{~nm}$ using the second harmonic of a Nd:YAG (yttrium aluminum garnet) laser (Spectra-Physics, $8 \mathrm{~ns}$ pulse width). Standard backscattering configuration of DFMW was used for the experiment. In nanosecond regime usually thermal effect dominates. Here we are interested mainly in the nonlinearity, which arises from the electronic response. Therefore polar-

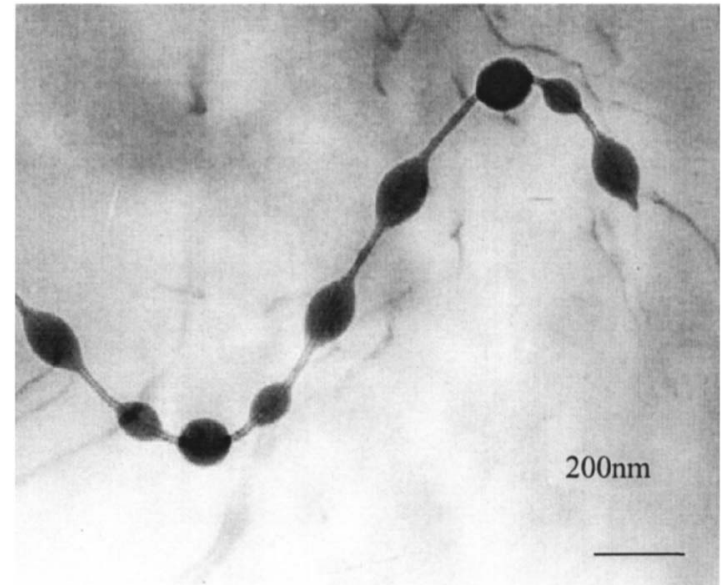

FIG. 2. TEM image of POTABQ-MWNTs

izations of the beams were chosen so as to avoid thermal effects. ${ }^{32}$ The two pump beams were vertically polarized and probe beam was horizontally polarized. Since crosspolarized beams do not interfere, they will not produce the thermal gratings which can give rise to additional FWM effect. In the present phase conjugation setup the pump-probe angle was adjusted to be nearly $8^{\circ}$. The two pump beams were of nearly equal intensity. Pump-probe intensity ratio was about 5 . The phase conjugate beam, which retraces the path of probe beam, was separated using a beam splitter. The experimental setup for DFMW was calibrated by measuring the ratio of $\chi^{(3)}$ ${ }_{\text {yxyx }}$ to $\chi^{(3)}{ }_{\text {xxxx }}$ for $\mathrm{CS}_{2}$. This ratio was found to be 0.688 , which is very close to the literature value $0.706 .^{33}$ The interacting beams had a diameter of nearly $8 \mathrm{~mm}$ and these were used for experiment without focusing.

In the open aperture and closed aperture $Z$-scan experiments the transmittance of the sample is measured as the sample is moved along the propagation direction $(z)$ of a focused Gaussian laser beam. In the present experiment the laser beam was focused with a lens of a focal length of $20 \mathrm{~cm}$ producing a beam waist $\omega_{0}$ of $13 \mu \mathrm{m}$ and a Rayleigh range $z_{0}$ of $1.5 \mathrm{~mm}$ in the sample. The sample was taken in a cuvette of path length of $1 \mathrm{~mm}$, which is less than the Rayleigh range in the sample. Therefore thin sample approximation is reasonably valid. The pulse was divided by a beam splitter into two beams. The reflected part was taken as the reference representing the incident pulse energy, and the transmitted beam was focused through the sample. The incident and the transmitted pulse energies were measured simultaneously by two pyroelectric detectors (Laser Precision Corporation, USA, RjP-735). Their values were collected by an energy ratiometer (Laser Projection Rj7620). The optical limiting measurements were carried out when the sample was fixed at the focal point.

\section{RESULTS AND DISCUSSION}

\section{A. The morphology}

Figure 2 shows a micrograph of POTABQ-MWNTs at a magnification of 50000 , which exhibits a bead-shaped struc- 


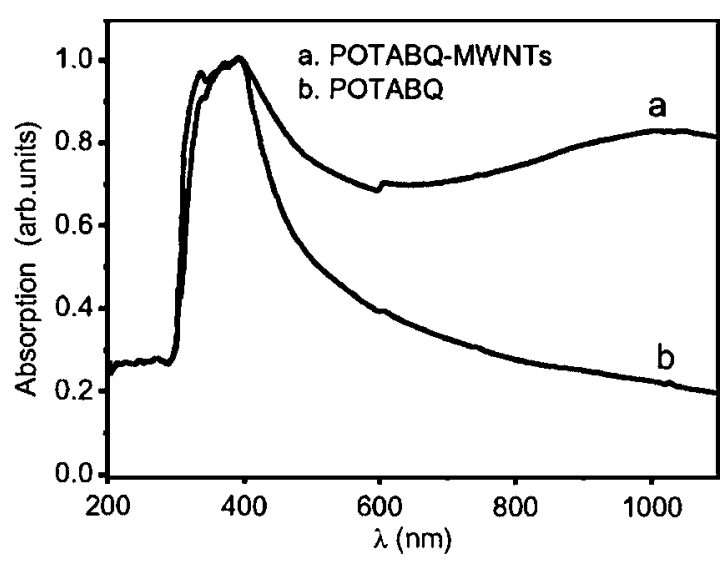

FIG. 3. Absorption spectra of POTABQ-MWNTs and POTABQ.

ture. Extensive microscopy studies have shown that the diameter of polymer cluster is about $100 \mathrm{~nm}$, while the diameter of tubes is about $10 \mathrm{~nm}$.

Tang and $\mathrm{Xu}^{22}$ showed a typical image of PPA-wrapped MWNTs. They considered that these tubes were chemically linked to the polymer. The initiators absorbed on the surface of carbon nanotubes led to the polymerization of monomer molecules. Thus the polymer chain could then wrap the whole MWNT. In our previous study, ${ }^{34}$ well-aligned polyaniline/carbon-nanotube composite (PANI-MWNT) films with strong molecular interactions were prepared by in situ aniline polymerization. Aligned MWNT films were encapsulated in the cores of the growing polymer chains, resulting in the formation of a PANI-MWNT hybrid material.

In the case of POTABQ-MWNTs, the mechanism of wrapping is different. Carboxyl is introduced to both the tips and the sidewall of the tubes, when MWNTs are refluxed in $\mathrm{HNO}_{3}$. The carboxyl is then converted to acyl chlorine group. POTABQ is grafted to the tubes via amide reaction of $-\mathrm{NH}_{2}$ group and acyl chlorine group. The morphology depends on the function group density on the tubes. Low acyl chlorine group density may lead to the formation of " beadshaped structure," while high acyl chlorine group density may lead to whole wrapping.

\section{B. UV-vis absorption spectra}

Figure 3 shows the UV-vis absorption spectra of POTABQ and POTABQ-MWNTs in room-temperature chloroform. POTAB solution exhibits an absorption band around $400 \mathrm{~nm}$ and a broad weak decaying tail in visible region. The absorption band in ultraviolet region ranging from 300 to $450 \mathrm{~nm}$ is assigned to the $\pi-\pi^{*}$ transition of aromatic thiophene ring in the main chain and benzene ring in the side chain. The broad weak decaying tial covering entire visible region arises from the $\pi-\pi^{*}$ transition of methine bridge. POTABQ-MWNT solution exhibits a strong absorption band around $400 \mathrm{~nm}$ and a broad absorption band ranging from 800 to $1100 \mathrm{~nm}$. The latter indicates that there is a significant ground-state interaction between POTABQ and MWNTs. Since POTABQ and MWNTs are joined through the $\pi-\pi$ and $p-\pi$ conjugated bonds, the resulting POTABQMWNT composites exhibit larger conjugation extend and
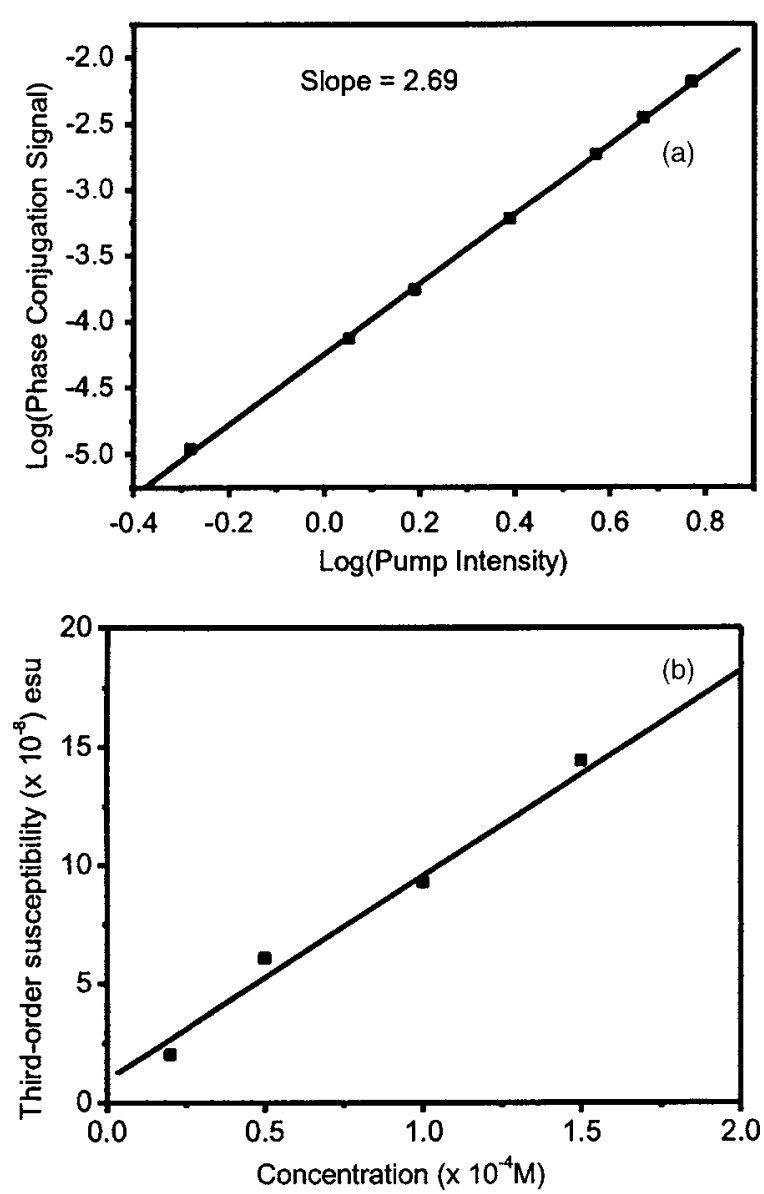

FIG. 4. (a) Log-log plot of phase conjugate signal intensity against pump beam intensity (in arbitrary units). (b) Concentration dependence of thirdorder nonlinearity for the same sample.

charge delocation. Similar phenomena were observed in NT/ PPA composites ${ }^{22}$ and P3OT-dye-SWNT composites. ${ }^{35}$

\section{DFMW measurements}

The POTABQ-MWNT and POTABQ solutions in chloroform were used. Pump beam energy was varied from 0.5 to $3 \mathrm{~mJ}$. All the experiments are carried out with samples having a concentration in the range of $10^{-4}-10^{-5} \mathrm{M}$. The compounds remain stable even after exposure to laser pulses for a long period of time, which is confirmed from absorption spectra recorded before and after the measurements. Figure 4(a) shows the log-log plot of the phase conjugate intensity against pump beam intensity. The plot is a straight line. This value may be considered as close to three and hence it is indicative of the third-order process involved in the phenomenon. Figure 4(b) shows the linear dependence of $\chi^{(3)}$ to the sample concentration for POTABQ-MWNTs. The $\chi^{(3)}$ was calculated with respect to a reference sample, $\mathrm{CS}_{2}$, using Eq. (1)

$$
\chi^{(3)}=\frac{l_{\mathrm{ref}}}{l}\left(\frac{n}{n_{\mathrm{ref}}}\right) \frac{\alpha l}{\left(1-e^{-\alpha l}\right) e^{-\alpha / 2}}\left(\frac{I}{I_{\mathrm{ref}}}\right) \chi_{\mathrm{ref}}^{(3)},
$$

where $l, n, \alpha$, and $I$ are the interaction length, the index of refraction, the absorption coefficient, and the DFWM signal intensity, respectively. The corresponding value of $\chi^{(3)}$ ref was 
TABLE I. Third-order NLO properties for POTABQ-MWNTs and POTABQ.

\begin{tabular}{ccccc}
\hline \hline Sample & $\chi^{(3)}\left(\times 10^{-9} \mathrm{esu}\right)$ & $\chi^{(3)} / \alpha\left(\times 10^{-13} \mathrm{esu}\right)$ & $\chi_{\text {MWNT }-p}^{(3)}\left(\times 10^{-9} \mathrm{esu}\right)$ & $\gamma\left(\times 10^{-31} \mathrm{esu}\right)$ \\
\hline POTABQ & $6.22 \pm 0.74$ & $6.28 \pm 0.75$ & $\ldots$ & $\gamma=98.60 \pm 11.73$ \\
POTABQ-MWNTs & $144.12 \pm 26.94$ & $93.58 \pm 17.49$ & $137.90 \pm 26.94$ & $\gamma_{c}=51.95 \pm 9.71$ \\
\hline \hline
\end{tabular}

$1.7 \times 10^{-12}$ esu (Ref. 36) and in our experimental conditions $I_{\text {ref }}=1 \mathrm{~mm}$ and $n_{\text {ref }}=1.33$ for nanosecond pulses. For the POTABQ-MWNT samples used in the measurements data in Eq. (1) were $l=1 \mathrm{~mm}, n=1.69$, and $\alpha=1.54 \times 10^{4} \mathrm{~cm}^{-1}$.

We can define figure of merit $F=\chi^{(3)} / \alpha$ for third-order nonlinearity which characterizes the nonlinear response that can be achieved for a given absorption loss. $\dot{F}$ values are very often used for comparing different types of absorbing samples. Measurement of DFWM gives combined contribution of POTABQ and MWNTs and photoinduced chargetransfer effect to nonlinearity. The third-order susceptibility for POTABQ $\chi^{(3)}$ POTABQ is measured to be $(6.22 \pm 0.74)$ $\times 10^{-9}$ esu as shown in Table I, while the third-order susceptibility for MWNTs $\chi^{(3)}{ }_{\text {MWNT }}(532 \mathrm{~nm}$, nanosecond) is only $0.309 \times 10^{-11} \mathrm{esu}$, as reported by Jin et al. ${ }^{12}$ The contribution of photoinduced charge transfer to third-order NLO response $\chi_{\text {PICT }}^{(3)}$ is then calculated to be $(137.90 \pm 26.94) \times 10^{-7}$ esu for POTABQ-MWNTs. The value of $\chi^{(3)}{ }_{\text {PICT }}$ is several orders of magnitude larger than that of $\chi_{\text {POTABQ }}^{(3)}$ and $\chi_{\text {MWNT }}^{(3)}$, which indicates that photoinduced charge-transfer effect enhanced the NLO response considerably. For convenience, we define $\chi_{\text {MWNT- } p}^{(3)}=\chi_{\text {MWNT }}^{(3)}+\chi_{\text {PICT }}^{(3)}$ Because the MWNTs we used have a wide distribution of lengths and diameters, it is difficult to assign directly the magnitude of $\gamma$ for the MWNT. However, we may calculate the average contribution from each carbon atom in the MWNTs using the following equation:

$$
\gamma=\chi^{(3)} / N L
$$

where $N$ is the number density of carbon atoms in our sample and $\chi^{(3)}$ is the purity of MWNTs, while $L$ is the local-field correction given by

$$
L=\left[\left(2+n^{2}\right) / 3\right]^{4} \text {. }
$$

By assuming a CNT number density of $10^{14} \mathrm{~cm}^{-3}$ it turns that $N=8 \pm 4 \times 10^{21} \mathrm{~cm}^{-3} .{ }^{13}$ The magnitude of $\gamma_{\text {C-MWNT-p }}$ for each atom is then calculated to be 5.20 $\times 10^{-30}$ esu for POTABQ-MWNTs. Therefore, the contribution of one carbon atom in the carbon nanotubes for POTABQ-MWNTs is three to four orders of magnitude larger than that of CNTs reported by Wang et al. ${ }^{11}$ and Botti et al. ${ }^{13}$ This is mainly due to the formation of intramolecular charge-transfer system for POTABQ-MWNTs and thus enlarge the conjugation extend and charge delocation, which is in accord with the previous discussed UV-vis spectra.

The measured values of the third-order nonlinear susceptibility, figure of merit, and the second-order hyperpolarizabilities of the sample are given in Table I.

\section{Z-scan measurements}

The open aperture of Z-scan experiments was used to measure the total transmittance through the samples. It is a measure of the overall nonlinear absorption, which may include excited-state absorption (ESA) as well as instantaneous two-photon absorption (ITPA). Effective nonlinear absorption coefficient $\beta_{\text {eff }}$ was calculated from open aperture $Z$-scan experiment by fitting the experimental data to the normalized transmittance. ${ }^{37}$

The normalized transmittance as a function of position $z$, $T_{\text {Norm }}(z)$, is given by

$$
T_{\text {Norm }}(z)=\frac{\ln \left[1+q_{0}(z)\right]}{q_{0}(z)},
$$

where $q_{0}(z)$ is given by

$$
q_{0}(z)=\frac{q_{00}}{1+\left(z / z_{0}\right)^{2}} .
$$

$z_{0}$ is the diffraction length of the beam, and $q_{00}$ $=\beta_{\text {eff }} I_{0} L_{\text {eff }}$, where $L_{\text {eff }}=[1-\exp (-\alpha L)] / \alpha . \beta_{\text {eff }}$ is the effective intensity-dependent nonlinear absorption coefficient and $I_{0}$ is the intensity of the light at the focus. $L_{\text {eff }}$ is known as the effective length of the sample defined in terms of the linear absorption coefficient $\alpha$ and the true optical path length through the sample $L$.

The closed aperture Z-scan was used to measure the nonlinear refraction. ${ }^{38}$ An easily measurable quantity $\Delta T_{p-v}$ is defined as the difference between the normalized peak and valley transmittances. The variation of $\Delta T_{p-v}$ is expressed as $\Delta T_{p-v}=0.406(1-S)^{0.25}$, for $\left|\Delta \Phi_{0}\right|<\pi$, where $\left|\Delta \Phi_{0}\right|$ $=(2 \pi / \lambda) L_{\text {eff }} \Delta n_{0}$ with $\Delta n_{0}=n_{2} I_{0}$, and $S$ is the linear transmittance of a far field aperture ( $S=0.1$ in this work). Experimentally, we determine $\Delta T_{p-v}$ from the normalized transmittance data, then we divide this value by $0.406(1$ $-S)^{0.25}(2 \pi / \lambda) I_{0} L_{\text {eff }}$ to obtain,

$$
n_{2}=\frac{\Delta n_{0}}{I_{0}}=\frac{\Delta T_{p-v}}{0.406(1-S)^{0.25}(2 \pi / \lambda) I_{0} L_{\text {eff }}} .
$$

Figure 5(a) shows a typical open aperture Z-scan, for POTABQ-MWNT and POTABQ solutions in roomtemperature chloroform. Decrease of transmission around the focal point is indicative of the presence of nonlinear absorption. The effective absorption coefficient $\beta_{\text {eff }}$ for POTABQMWNTs and POTABQ are $\left(1.59 \pm 0.18 \times 10^{-8}\right)$ and $\left(1.33 \pm 0.12 \times 10^{-9}\right) \mathrm{mW}^{-1}$, respectively. Nonlinear absorption under nanosecond excitation for POTABQ-MWNTs can be easily explained using a five-level model involving $S_{0}, S_{1}$, $S_{2}, T_{1}$, and $T_{2}$, as shown in Fig. $6 .{ }^{39}$ Here $S_{\mathrm{s}}^{\prime}$ and $T_{\mathrm{s}}^{\prime}$ correspond to singlet and triplet manifolds, each containing a number of vibrational levels. Molecules are initially excited 

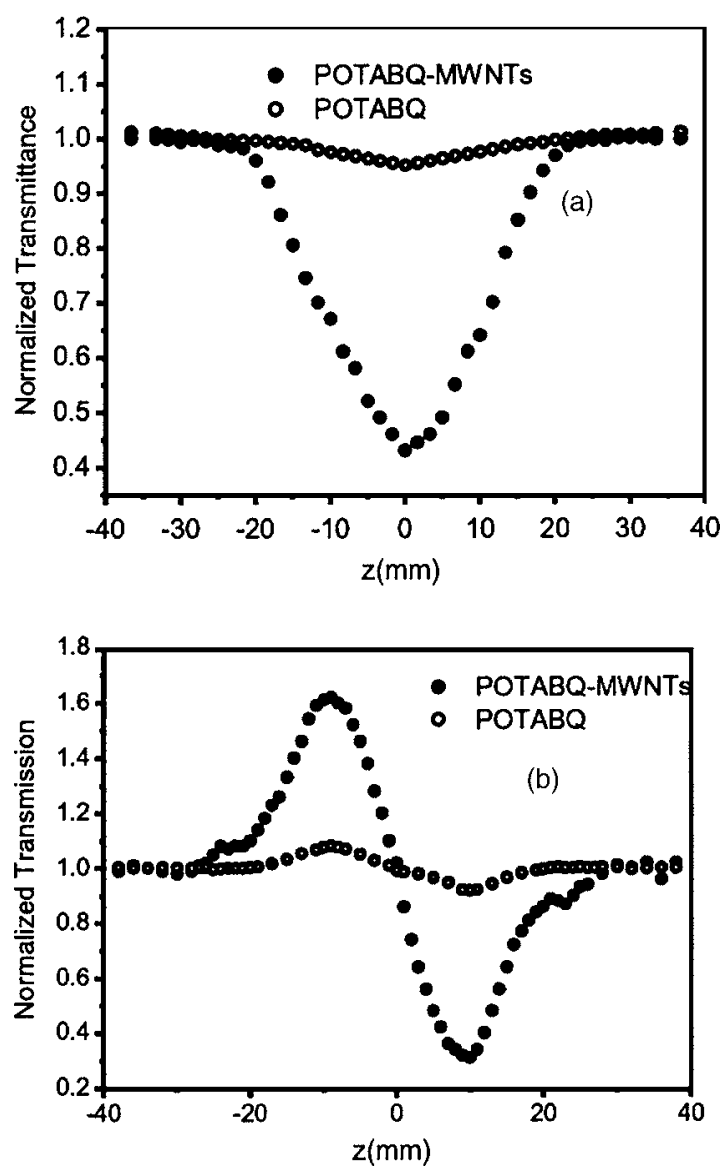

FIG. 5. (a) Open aperture and (b) closed aperture Z-scan curves for POTABQ-MWNTs and POTABQ.

electronically from lowest vibrational level of $S_{0}$ to upper vibrational levels of $S_{1}\left[S_{1} \leftarrow S_{0}(\nu=0)\right]$. Here $\nu$ corresponds to vibrational quantum number. From there molecules will arrive at lowest vibrational level of $S_{1}(\nu=0)$ in picoseconds by nonradiative decay. In nanosecond time scale $\left(S_{2} \leftarrow S_{1}\right)$ singlet transition does not deplete population on $S_{1}$ appreciably as atoms excited to $S_{2}$ decay to $S_{1}$ itself within picoseconds. From $S_{1}$ molecules finally go to $T_{1}$ via intersystem crossing wherefrom they are excited to $T_{2}$. Nonlinear absorption observed here is a consequence of $T_{2} \leftarrow T_{1}$ absorption $\left[T_{2} \leftarrow T_{1}(\nu=0)\right]$. The last two processes $\left(S_{2} \leftarrow S_{1}\right.$ and $T_{2}$ $\left.\leftarrow T_{1}\right)$ are known as ESA, and if their cross sections are larger than that of the ground-state linear absorption, then

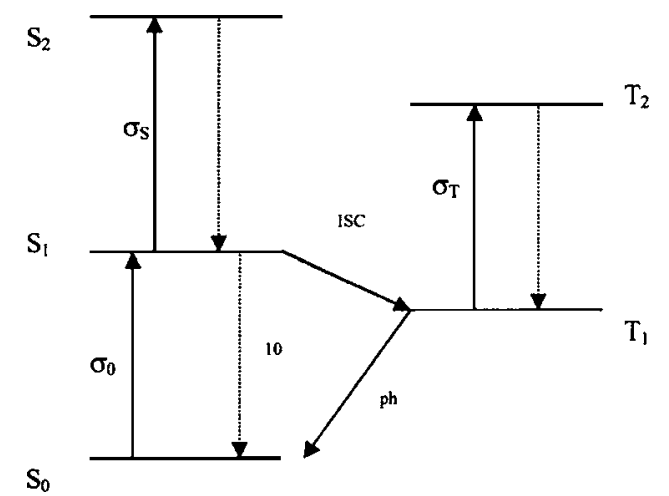

FIG. 6. Energy-level diagram (five-level model) $\left(T_{1} \leftarrow S_{1}\right.$ is nonradiative). these are referred to as reverse saturable absorption (RSA). We attribute the observed nonlinear absorption to strong ESA or RSA. Typical peak intensities at the focus are in the range of $10^{8}-10^{9} \mathrm{~W} / \mathrm{cm}^{2}$.

Figure 5(b) shows the closed aperture $Z$-scan for POTABQ-MWNT and POTABQ solutions in roomtemperature chloroform. Both samples are found to exhibit peak-to-valley characteristic, indicating the self-defocusing property, which is represented as a negative third-order nonlinear refractive index $\left(n_{2}\right)$. Because of the near-resonant excitation this negative nonlinearity, in the nanosecond time scales, could be due to the thermal excitation of the medium. Figure 5(b) illustrates the normalized transmittances at various irradiances of our closed aperture $Z$-scans, from which $\Delta T_{p-v}$ can be measured directly, We obtain $n_{2}$ $=(2.17 \pm 0.29) \times 10^{-15} \mathrm{~m}^{2} \mathrm{~W}^{-1}$ for POTABQ-MWNT solution and $n_{2}=(9.37 \pm 0.62) \times 10^{-17} \mathrm{~m}^{2} \mathrm{~W}^{-1}$ for POTABQ solution.

In general, it is very difficult to quantify the individual contributions of various channels (real and imaginary parts, thermal part, due to excited states, etc.) to a nonlinear signal. To the extent possible, we have tried to separate the thermal and electronic nonlinear mechanisms. This has been achieved by cross polarizing the grating forming beams in DFWM, whereby the thermal mechanism is suppressed and we measured only the electronic nonlinearity. The open aperture Z-scan shows a third-order nonlinear absorption mechanism where ESA or RSA is the dominating extinction mechanism. However, the determination of the contributions of excited states demands knowledge of excited-state cross sections and lifetimes. We are in the process of doing the spectroscopy of these states using picosecond and femtosecond time-resolved pump-probe, four-wave mixing, and $Z$-scan experiments and will publish the results and their implications on nonlinearity in the near future. The closed aperture $Z$-scan study provides evidences of electronic contributions to the nonlinear response of POTABQ-MWNT solutions, which has been previously reported by Jin et al. ${ }^{12}$ and Vivien et al. ${ }^{40}$ for polymer/nanotube composite materials irradiated with nanosecond pulses.

\section{E. Optical limiting properties and mechanistic implications}

Optical limiting occurs when the optical transmission of a material decreases with increasing laser fluence, a property that is desirable for protection of sensors and human eyes from intense laser radiation. Several nonlinear effects lead to optical limiting: nonlinear absorption (reverse saturable absorption $^{41}$ and multiphotonics absorption ${ }^{42}$ ), nonlinear refraction (electronics ${ }^{43}$ or thermal effects ${ }^{44}$ ), and nonlinear scattering (solvent bubble formation and/or particle sublimation ${ }^{45-47}$ or mismatched indices ${ }^{48}$ ).

Figure 7 shows the optical limiting responses for POTABQ-MWNTs and POTABQ. The on-focus data are plotted to summarize the overall linear and nonlinear optical extinction in both samples. In this plot the straight lines indicate the linear transmittance in the absence of nonlinear contributions. When a chloroform solution of POTABQ 


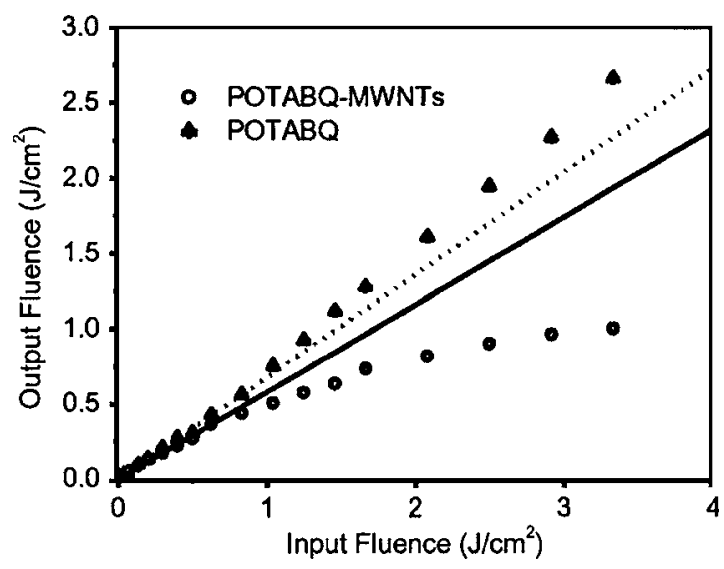

FIG. 7. Optical limiting responses for POTABQ-MWNT and POTABQ solutions in room-temperature chloroform.

$(1.0 \mathrm{mg} / \mathrm{mL})$ is irradiated by the $8-\mathrm{ns}$ pulses of $532-\mathrm{nm}$ laser light, the transmitted fluence linearly increases in the region of low incident fluence (linear transmittance is 68\%). The output starts and continues to deviate from the lineartransmission line from the input of ca. $1.2 \mathrm{~J} / \mathrm{cm}^{2}$, implying that the intense illumination gradually bleaches the POTABQ to transparency, probably by the laser-induced photolysis of the polymer chain. POTABQ-MWNT solutions respond to the optical pluse in a strikingly different way. It can be clearly seen that POTQABQ-MWNTs has enhanced optical extinctions with increasing fluence as the data for the sample can be seen to dip further below its linear transmittance line as the incident fluence increases. The linear transmittance of a dilute solution of POTABQ-MWNTs $(0.5 \mathrm{mg} / \mathrm{mL})$ is only $58 \%$, although its concentration is half of the parent POTABQ, probably because of the optical losses caused by nanotube absorption and scattering. As the incident fluence increases, the POTABQ-MWNT solution becomes opaque, instead of transparent, with its transmitted fluence eventually level off or saturating at $1.66 \mathrm{~J} / \mathrm{cm}^{2}$ (saturation fluence). Clearly, nanotubes endowed the POTABQ-MWNTs with limiting power. While the POTABQ is liable to photolysis, the POTABQ-MWNTs are stable at very high incident fluence. The energy sinking and radical trapping functions or aromatic rings often protect polymer molecules from photodegradation, ${ }^{49}$ and the extensively conjugated graphite aromatic system of the nanotubes may have enhanced the resistance of the POTABQ chains against the harsh laser irradiation.

The optical limiting mechanisms for carbon nanotubes/ polymer solutions or films are rather complicated and quite different from that of carbon nanotubes suspensions. Sun et $a .^{50}$ and Vivien et $a l^{40}$ reported the first studies of MWNT and SWNT suspensions, respectively, and compared their optical limiting performance with those of carbon black suspensions $(\mathrm{CBS})$ and fullerence $\mathrm{C}_{60}$ carbon nanotubes were shown to exhibit nonlinear behaviors very different to $\mathrm{C}_{60}$ (reverse saturable absorption), but very similar to those of CBS, i.e., nonlinear scattering due to bubble growth and plasma formation. Tang et al. ${ }^{22}$ performed standard optical limiting measurements at $532 \mathrm{~nm}$ using PPA/MWNT homogeneous solutions, the optical limiting responses are depen- dent on the nanotube content in the samples. Jin et al. ${ }^{12}$ reported the optical limiting properties of polymer-coated and polymer-grafted MWNTs. The polymers they used are largely colorless and do not significantly interfere with the ground-state absorption of the nanotubes. However, they did not give any mechanism study of the optical limiting responses for polymer/MWNT composites. Riggs et al. $^{51}$ reported on mechanistic differences in the origin of nonlinear dissipation of laser pulses at $532 \mathrm{~nm}$ in suspended and soluble MWNTs and SWNTs. They solubilized shortened carbon nanotubes by attaching them to poly(propionylethylenimine-co-ethylenimine) (PPEI-EI) or by functionalizing the nanotubes with octadecylamine. The soluble carbon nanotubes then formed homogeneous solutions in chloroform at room temperature. Essentially they concluded that the nonlinear extinction mechanism was principally nonlinear scattering in the aqueous suspensions of MWNTs, while a RSA process in the solubilized systems. A RSA process requires the existence of a photo-excited state with an absorption cross section exceeding that of the ground-state absorption cross section. O'Flaherty et al. ${ }^{23}$ carried out a comprehensive study on NLO properties of PmPV/ MWNT films. They attributed the optical limiting mechanism to the combination of nonlinear scattering, nonlinear absorption (probably RSA), and nonlinear refraction (electronic contributions).

For a better understanding of the origin of optical limiting in POTABQ-MWNTs, both DFWM and Z-scan experiments have been carried out to give a comprehensive study on its nonlinear optical properties. The $Z$-scan technique is a sensitive and simple characterization method of the intensitydependent optical properties of materials (nonlinear absorption, refraction or nonlinear scattering). Z-scan experiments have been carried out to dissociate nonlinear refraction effects from nonlinear absorption and scattering contributions. With an open aperture, one usually only observes a dip in the transmission curve, which can be the signature of either nonlinear absorption or nonlinear scattering. With a closed aperture, two mechanisms can be possibly observed, nonlinear absorption and nonlinear refraction. In order to determine the physical origin of nonlinear scattering, Vivien et al. ${ }^{40}$ have carried out pump-probe experiments at $532 \mathrm{~nm}$, with nanosecond pulses.

Based on the DFWM and Z-scan (open aperture and closed aperture) experiments shown before, the optical limiting mechanisms for POTABQ-MWNTs are assigned to the combination of nonlinear scattering, nonlinear absorption, and nonlinear refraction. According to the open aperture study [shown in Fig. 5(a)] and discussed using a five-level model (shown in Fig. 6) before, the dominant nonlinear absorption mechanism for POTABQ-MWNTs is ESA or RSA (when the cross section of excited state exceeding that of the ground state). The evidence of nonlinear refraction contributions to the NLO and optical limiting responses for POTABQ-MWNTs is given by closed aperture Z-scan [shown in Fig. 5(b)]. The nonlinear scattering process for POTABQ-MWNTs has not been studied mainly due to lacking of the relative experiment setup and skills. However, according to the study of O'Flaherty et $a .^{23}$ for PmPV/ 
MWNT composites, the sample with higher MWNTs (5.9\%) exhibits the characteristic of Mie scattering, which has previous reported in CBS and carbon nanotubes. The scattered signal at every angle increases with the MWNT content increasing. Since the MWNT content for our POTABQMWNT sample is high (about 7\%), we suppose that nonlinear scattering occurs and plays important role in optical limiting performance for this material. We are going to carry out this study in the near future to elucidate the nonlinear optical mechanism for POTABQ-MWNT composites.

\section{CONCLUSIONS}

Strong phase conjugate reflections were observed from the solutions of POTABQ-MWNTs under DFWM configuration. Third-order NLO susceptibility, second-order hyperpolarizability, and figure of merit were calculated for the sample at $532 \mathrm{~nm}$. Effective nonlinear absorption coefficient $\beta_{\text {eff }}$ and nonlinear refraction $n_{2}$ were obtained using open aperture and close aperture $Z$-scan techniques at the same wavelength, respectively. Optical limiting property of the sample was also investigated and its mechanism was discussed.

\section{ACKNOWLEDGMENTS}

This work was supported by the National Natural Science Foundation of China (Grant No. 60307001) and the Natural Science Foundation of Tianjin City.

${ }^{1}$ M. Bass, J. M. Enoch, E. W. V. Stryland, and W. L. Wolfe, Handbook of Optics IV, Fiber Optics and Nonlinear Optics, 2nd ed. (McGraw-Hill, New York, 2001).

${ }^{2}$ G. L. Stegeman and W. E. Torruellas, Philos. Trans. R. Soc. London, Ser. A 354, 645 (1996).

${ }^{3}$ C. N. R. Rao, B. C. Satishkumar, A. Govindaraj, and M. Nath, ChemPhysChem 2, 78 (2001).

${ }^{4}$ P. Fournet, J. N. Coleman, B. Lahr, A. Drury, W. J. Blau, D. F. O’Brien, and H. H. Horhold, J. Appl. Phys. 90, 969 (2001).

${ }^{5}$ R. Xie and J. Jiang, J. Appl. Phys. 83, 3001 (1998).

${ }^{6}$ R. Xie and J. Jiang, Chem. Phys. Lett. 280, 66 (1997).

${ }^{7}$ R. Xie and J. Jiang, Appl. Phys. Lett. 71, 1029 (1997).

${ }^{8}$ V. Margulis and T. Sizikova, Physica B 245, 173 (1998).

${ }^{9}$ X. Wan, J. Dong, and D. Xing, Phys. Rev. B 58, 6756 (1998).

${ }^{10}$ X. Liu et al., Appl. Phys. Lett. 74, 164 (1999).

${ }^{11}$ S. F. Wang, W. T. Huang, H. Yang, Q. H. Gong, Z. J. Shi, X. H. Zhou, D. Qiang, and Z. N. Gu, Chem. Phys. Lett. 320, 411 (2000).

${ }^{12}$ Z. X. Jin, X. Sun, G. Q. Xu, S. H. Goh, and W. Ji, Chem. Phys. Lett. 318, $505(2000)$.

${ }^{13}$ S. Botti, R. Ciardi, L. De Dominicis, L. S. Asilyan 1, R. Fantoni, and T. Marolo, Chem. Phys. Lett. 378, 117 (2003).

${ }^{14}$ A. G. Rozhin, Y. Sakakibara, and M. Tokumoto, Thin Solid Films 46465, 368 (2004).

${ }^{15}$ Z. W. Wang, C. L. Liu, and H. Xiang, J. Phys. D 37, 1079 (2004).

${ }^{16}$ D. A. Akimov, M. V. Alfimov, and S. O. Konorov, J. Exp. Theor. Phys.
98, 220 (2004).

${ }^{17}$ N. S. Sariciftci, L. Smilowitz, A. J. Heeger, and F. Wudl, Science 258, 1474 (1992)

${ }^{18}$ S. Morita, A. A. Zakhidov, and K. Yoshino, Solid State Commun. 82, 249 (1992).

${ }^{19}$ K. Yoshino, X. H. Yin, S. Morita, T. Kawai, and A. A. Zakhidov, Chem. Express 7, 817 (1992).

${ }^{20} \mathrm{~K}$. Yoshino, X. H. Yin, S. Morita, T. Kawai, and A. A. Zakhidov, Solid State Commun. 85, 85 (1993).

${ }^{21}$ E. S. Maniloff, D. Vacar, D. McBranch, H. L. Wang, and A. J. Heeger, Opt. Commun. 141, 243 (1997).

${ }^{22}$ B. Z. Tang and H. Y. Xu, Macromolecules 32, 2569 (1999).

${ }^{23}$ S. M. O'Flaherty, S. V. Hold, M. E. Brennan, M. Cadek, A. Drury, J. N. Coleman, and W. J. Blau, J. Opt. Soc. Am. B 20, 49 (2003).

${ }^{24}$ S. A. Jenkhe, Nature (London) 322, 345 (1986).

${ }^{25}$ W. C. Chen and S. A. Jenkhe, Macromolecules 28, 454 (1995).

${ }^{26}$ W. C. Chen and S. A. Jenkhe, Macromolecules 28, 465 (1995).

${ }^{27}$ S. A. Jenkhe and S. K. Lo, Appl. Phys. Lett. 54, 2524 (1989).

${ }^{28}$ S. A. Jenkhe and W. C. Chen, Appl. Phys. Lett. 57, 126 (1990).

${ }^{29}$ W. H. Yi, W. Feng, M. Cao, and H. C. Wu, Polym. Adv. Technol. 15, 431 (2004).

${ }^{30}$ W. H. Yi, C. Y. Li, J. B. Zheng, H. C. Wu, and Z. G. Zhang, Acta Photonica Sin. 32, 632 (2003).

${ }^{31}$ W. H. Yi, W. Feng, Y. L. Xu, and H. C. Wu, Jpn. J. Appl. Phys., Part 1 44, 3022 (2005).

${ }^{32}$ D. J. McGraw, A. E. Seigman, G. M. Wallraff, and R. D. Miller, Appl. Phys. Lett. 54, 1713 (1989).

${ }^{33}$ R. A. Fisher, Optical Phase Conjugation (Academic, New York, 1983).

${ }^{34}$ W. Feng, X. D. Bai, Y. Q. Lian, J. Liang, X. G. Wang, and K. Yoshino, Carbon 41, 1551 (2003).

${ }^{35}$ S. Bhattacharyya, E. Kymakis, and G. A. J. Amaratunga, Chem. Mater. 16, 4819 (2004).

${ }^{36}$ S. V. Rao, N. K. M. N. Srinivas, D. N. Rao, L. Giribabu, B. G. Maiya, R. Philip, and G. R. Kumar, Opt. Commun. 182, 255 (2000).

${ }^{37}$ M. Sheik Bahae, A. A. Said, T. H. Wei, D. J. Hagan, and E. W. V. Stryland, IEEE J. Quantum Electron. 26, 760 (1990).

${ }^{38}$ F. E. Hernandez, A. Marcano, O. Ysaias Alvarado, A. Biondi, and $\mathrm{H}$. Maillotte, Opt. Commun. 152, 77 (1998).

${ }^{39}$ J. Callaghan, W. J. Blau, and F. Z. Henari, J. Nonlinear Opt. Phys. Mater. 19, 505 (2000).

${ }^{40}$ L. Vivien et al., Chem. Phys. Lett. 307, 317 (1999).

${ }^{41}$ J. W. Perry, in Nonlinear Optics of Organic Molecules and Polymers, edited by H. S. Nalwa and S. Miyata (CRC, New York, 1997).

${ }^{42}$ A. A. Said, M. Sheik-Bahae, D. J. Hagan, T. H. Wei, J. Wang, and E. W. Van Stryland, J. Opt. Soc. Am. B 9, 405 (1992).

${ }^{43}$ R. W. Boyd, Nonlinear Optics (Academic, New York, 1992).

${ }^{44}$ B. L. Justus, A. L. Huston, and A. J. Campillo, Appl. Phys. Lett. 63, 1483 (1993).

${ }^{45}$ K. J. McEwan, P. K. Milsom, and D. B. James, Proc. SPIE 3472, 42 (1998).

${ }^{46}$ K. M. Nashold and W. D. Powell, J. Opt. Soc. Am. B 12, 1228 (1995).

${ }^{47}$ K. Mansour, M. J. Soileau, and E. W. Van Stryland, J. Opt. Soc. Am. B 9, 1100 (1992).

${ }^{48}$ V. Joudrier, P. Bourdon, F. Hache, and C. Flytzanis, Appl. Phys. B 70, 105 (2000).

${ }^{49}$ G. Scott, Mechanism of Polymer Degradation and Stabilization (Elsevier, London, 1990).

${ }^{50}$ X. Sun, R. Q. Yu, G. Q. Xu, T. S. A. Hor, and W. Ji, Appl. Phys. Lett. 73, 3632 (1998).

${ }^{51}$ J. E. Riggs, D. B. Walker, D. L. Carrol, and Y. P. Sun, J. Phys. Chem. B 104, 7071 (2000). 